L. Belabed - G. Senon - M.-C. Blanc •

A. Paillard $\cdot$ L. Cynober $\cdot$ S. Darquy

\title{
The equivocal metabolic response to endotoxaemia in type 2 diabetic and obese ZDF rats
}

Received: 30 September 2005 / Accepted: 1 February 2006 / Published online: 19 April 2006

C) Springer-Verlag 2006

\begin{abstract}
Aims/hypothesis: The metabolic and endocrine disturbances associated with obesity and type 2 diabetes may impair the normal metabolic response to injury. Our objective was to investigate amino acid metabolism in endotoxaemic type 2 diabetic obese rats. Materials and methods: A metabolic study was performed over 4 days using male Zucker diabetic fatty (ZDF) rats $(f a / f a)$ and lean littermates $(f a /+)$ divided into three groups: ad libitum-fed groups which underwent no treatment, lipopolysaccharide (LPS)-treated groups receiving E. coli LPS by i.p. injection, and pair-fed groups to the respective LPS groups. We evaluated the effect of endotoxaemia on body weight, food intake and tissue weights. Nitrogen loss and muscular proteolysis were measured daily by determination of urinary 3-methylhistidine (3-MH) excretion. Plasma, intestine and muscle amino acid levels were measured. Results: The data showed that ad libitum-fed ZDF rats had lower plasma arginine and glutamine levels than ad libitum-fed control rats. Compared with control rats, the LPS-treated ZDF rats presented lower thymic involution, a lower 3-MH:creatinine ratio and higher cumulative nitro-
\end{abstract}

L. Belabed and G. Senon made an equal contribution to this study.

L. Belabed · G. Senon · M.-C. Blanc ·

A. Paillard · L. Cynober · S. Darquy

Laboratory of Biological Nutrition,

EA 2498, Paris Descartes University,

Paris, France

M.-C. Blanc · L. Cynober

Laboratory of Clinical Biochemistry A,

Hôtel-Dieu, AP-HP,

Paris, France

L. Belabed $(\bowtie)$

Laboratoire de Biologie de la Nutrition,

EA 2498, Faculté de Pharmacie, Université René Descartes,

4 avenue de l'Observatoire,

75270 Paris, France

e-mail: linda.belabed@nutrition-paris5.org

Tel.: +33-1-53739945

Fax: $+33-1-53739952$ gen balance. Conclusions/interpretation: Against our working hypothesis, ZDF rats did not show an impaired metabolic response, and even appeared to be less sensitive to the stress.

Keywords Amino acids $\cdot$ Arginine $\cdot$ E. coli lipopolysaccharide $\cdot$ Endotoxaemia $\cdot$ Metabolic response 3-methylhistidine $\cdot$ Nitric oxide $\cdot$ Nitrogen balance $\cdot$

Type 2 diabetes Zucker diabetic fatty rat

Abbreviations AL-C: ad libitum-fed control rats . AL-D: ad libitum-fed diabetic rats - BCAA: branchedchain amino acid - EDL: extensor digitorum longus (muscle) - LPS: lipopolysaccharide - LPS-C: LPS-treated control rats - LPS-D: LPS-treated diabetic rats ·

3-MH: 3-methylhistidine - NO: nitric oxide ·

NOx: NO-based by-products nitrite and nitrate $\cdot P F$ : pairfed - PF-C: PF control rats - PF-D: PF diabetic rats . PLSD: protected least significance difference .

ZDF: Zucker diabetic fatty (rat)

\section{Introduction}

Type 2 diabetes is commonly associated with obesity, which contributes to an increase in all-cause morbidity and mortality $[1,2]$. The overall prevalence of obesity has increased steadily over the last decade, leading to increasing numbers of obese patients requiring intensive care [1]. However, from a metabolic point of view, almost nothing is known as to how obese patients adapt to injury. One study [3] reported that morbidly obese patients face an eight-fold higher mortality following blunt trauma than non-obese patients. The metabolic response to injury (e.g. surgery, trauma, sepsis) plays a key role in the maintenance of vital functions and the promotion of the healing mechanisms. This process is characterised by an inflammatory response including cytokine production, which in turn induces a hypercatabolic state [4]. The metabolic response to stress in obese type 2 diabetic patients who are critically ill may be modified, since it occurs in a population with pre-existing 
metabolic and endocrine disturbances. In particular, the regulation of carbohydrate metabolism is altered, and it is well known that poorly controlled glycaemia is a major factor of mortality in intensive care unit patients [5]. These patients are characterised by fasting and postprandial hyperglycaemia, insulin resistance, and abnormally high hepatic glucose production in both fasted and fed states [6]. Diabetes affects protein metabolism, especially the regulation of protein synthesis and protein breakdown, which become altered [7]. It is therefore logical that such patients would have specific nutritional requirements.

An effective nutritional support strategy cannot be designated without careful evaluation of a potentially impaired metabolic response to stress. To date, very few studies [6] have been conducted on the metabolic response to injury in type 2 diabetic obese patients, especially in terms of amino acid metabolism. Furthermore, to the best of our knowledge, no experimental studies on the nutritional status of obese type 2 diabetic rats in a stress situation have yet been published. Our working hypothesis was that obesity may worsen the metabolic response to injury. The aim of this study was to characterise nutritional disturbances in response to endotoxaemia in a model of obese type 2 diabetic rats. We used the model of male Zucker diabetic fatty (ZDF) rats homozygous for nonfunctional leptin receptors $(f a / f a)$ and developing hyperphagia, obesity, hyperlipidaemia and hyperglycaemia [8, 9]. This rat strain is a recognised model mimicking human type 2 diabetes with impaired glucose tolerance associated with insulin resistance. Their littermates are heterozygous for normal receptors $(f a /+)$ and remain lean and normoglycaemic [9].

\section{Materials and methods}

Animals and research design

Animal care and experimentation complied with French regulatory requirements (French Ministry of Agriculture and Forestry authorisation). The study design was reviewed by the local ethics committee and one of the co-authors (L. Cynober) is authorised to use rodent models of stress (Authorisation No. 75-461).

Twelve-week-old male ZDF rats, which were designated as diabetic (D groups), and their lean littermates $(f a /+)$, which were designated as controls (C groups), were used (Charles River Laboratories, L'Arbresle, France). In this strain, hyperglycaemia appears at about 7 weeks of age, and all obese male rats are fully diabetic by 12 weeks of age (Charles River Laboratories nomenclature: ZDF falfa rats). The animals were housed individually in metabolic cages for a 3-day acclimatisation period, and body weight, food intake and volume of urine were recorded daily. They were housed in a temperature-controlled environment $\left(21 \pm 1^{\circ} \mathrm{C}\right)$ with a 12-h light-darkness cycle, and were fed a Purina 5008 diet ad libitum (composition, percent of total [in calories]: protein $23.5 \%$, fat, $17 \%$, carbohydrates $56 \%$ )
(IPS, Wellingborough, UK). All rats were allowed free access to deionised water.

After acclimatisation, C- and D-group animals were further randomised into three sets. On the one hand, we formed ad libitum-fed groups which underwent no treatment and lipopolysaccharide (LPS) groups receiving a single dose of Escherichia coli LPS (0127:B8 serotype; Sigma-Aldrich, France) at $3 \mathrm{mg} / \mathrm{kg}$ of body weight, by i.p. injection. Previous studies from our laboratory $[10,11]$ have shown that this dose of LPS induces a reproducible transient catabolic state without lethality, followed by recovery 3 days after injury. We also formed pair-fed (PF) groups to the respective LPS groups that received an i.p. injection of $300 \mu \mathrm{l} 0.9 \%$ sodium chloride. The rationale for the study of a PF group is that LPS induces anorexia [12]. Therefore, we used PF groups to separately study the effects of LPS treatment and the effects of restricted food consumption. Rats from PF groups received the same amount of food as the corresponding LPS group, but with a delay of $24 \mathrm{~h}$. Thus, there was a total of six rat groups: ad libitum-fed diabetic (AL-D, $n=7$ ), ad libitum-fed control (AL-C, $n=7$ ), LPS diabetic (LPS-D, $n=8$ ), LPS control (LPS-C, $n=7)$, PF diabetic (PF-D, $n=7$ ) and PF control (PF-C, $n=7$ ).

Three days after the different treatments, all 24-h-fasted rats were anaesthetised by isoflurane inhalation and then killed by decapitation. Blood samples were collected on sodium heparinate (Heparine Choay 25,000 IU/5 ml; Sanofi-Synthélabo, Vitry-sur-Seine, France).

Plasma insulin and glucose

Insulin was determined by RIA using an INSIK-5 Kit (Diasorin, Saluggia, Italy). Glycaemia was measured by a hexokinase endpoint method (Olympus AU600, Rungis, France).

\section{Plasma albumin}

In endotoxaemia, albuminaemia is a global marker of the inflammatory reaction [11]. Albumin was assayed by immunonephelometry using the Array System 360 analyser (Beckman-Coulter Instruments, Roissy, France). Rabbit IgG (Dako, Labarthe Inard, France) cross-reacting with rat albumin was used.

Tissue removal

\section{Thymus and spleen}

In experimental stress situations, thymic involution and hypertrophy of the spleen usually co-occur, thus indicating dysimmunity [11]. The thymus and spleen were rapidly removed and weighed immediately after killing. 


\section{Muscles}

Stress triggers a loss of muscle mass [11], and variations in the concentrations of certain amino acids are characteristic of the response to stress and/or insulin resistance [13]. The extensor digitorum longus (EDL) muscle was rapidly removed from the hindlimb, then weighed, frozen in liquid nitrogen and stored at $-80^{\circ} \mathrm{C}$ until analysis.

\section{Jejunum and ileum}

The intestine was resected from the ligament of Treitz to the ileocaecal junction to study amino acid levels from the intestinal compartment. The mucosa of the proximal parts of the jejunum and ileum was washed with $\mathrm{NaCl}(0.9 \%)$, everted and scraped, then weighed and rapidly frozen in liquid nitrogen, and stored at $-80^{\circ} \mathrm{C}$ until analysis.

\section{Liver}

The liver was removed, weighed and frozen in liquid nitrogen and stored at $-80^{\circ} \mathrm{C}$ until analysis.

\section{Urinary parameters}

The daily 24-h-collected urine samples were stored at $-20^{\circ} \mathrm{C}$ until dosage assay.

\section{Urinary 3-methylhistidine}

Urinary excretion of 3-methylhistidine (3-MH) provides one of the best estimates of myofibrillar protein catabolism in rat skeletal muscle. 3-MH is released during myofibrillar protein breakdown without being either metabolised or reutilised for protein synthesis [14]. Samples were hydrolysed with $\mathrm{HCl}(35 \%)$ at $100^{\circ} \mathrm{C}$ and then filtered before quantification by ion-exchange chromatography combined with ninhydrin detection (Hitachi L8500-A, Tokyo, Japan). Results are expressed in $\mu \mathrm{mol} 3-\mathrm{MH} / \mathrm{mmol}$ creatinine to take muscle mass into account in the evaluation of myofibrillar protein breakdown. Urinary creatinine was measured by the Jaffé reaction on an Olympus AU 600 analyser (Rungis, France) [15].

\section{Nitrogen balance}

Nitrogen balance corresponded to the difference between daily total nitrogen intake and daily urinary nitrogen output. Total nitrogen intake was calculated on the basis of $100 \mathrm{~g}$ Purina 5,008 diet supplying an estimated $23.5 \mathrm{~g}$ protein, with $100 \mathrm{~g}$ protein providing an estimated $16 \mathrm{~g}$ nitrogen. Thus, nitrogen intake $=$ food intake $\times 3.76 \%$. Urinary nitrogen $(\mathrm{g} / \mathrm{l})$ was quantified by chemiluminescence [16] using an Antek 9000 apparatus (Antek, Houston, TX, USA). Urinary nitrogen output was calculated using the following formula: urinary nitrogen $(\mathrm{g} / \mathrm{l}) \times 24$-h urinary volume (1).

\section{Urinary nitrate and nitrite}

Nitric oxide (NO) in the urine samples was evaluated by the well-recognised method based on indirect measurements of the NO-based by-products nitrite and nitrate $($ nitrite + nitrate $=\mathrm{NOx})$ via the Griess reaction [17] $(\mathrm{R} \& \mathrm{D}$ Systems, Lille, France). Urinary NOx output was calculated using the following formula: urinary $\mathrm{NOx}(\mathrm{nmol} /$ $\mathrm{ml}) \times 24$-h urinary volume $(\mathrm{ml})$.

Determination of amino acid concentration and total tissue protein

Plasma was deproteinised with a $30 \%(\mathrm{w} / \mathrm{v})$ sulfosalicylic acid solution. The supernatants were stored at $-80^{\circ} \mathrm{C}$ until amino acid analysis.

Frozen tissues (skeletal muscles, liver and intestinal mucosa) were homogenised in ice-cold $10 \%$ trichloroacetic acid containing $0.5 \mathrm{mmol} / 1$ EDTA. The pellets were treated with ethanol/ether, and the supernatants were stored at $-80^{\circ} \mathrm{C}$ until amino acid analysis.

Amino acids were separated and quantified by ion exchange chromatography (Aminotac-LC 500; Jeol, Croissy-sur-Seine, France).

\section{Data analysis}

Data are expressed as means \pm SEM. Statistical analysis was performed using Statview software. The parameters were analysed by one-way ANOVA followed by Fisher's protected least significance difference (PLSD) test. The two groups $(f a / f a)$ and $(f a /+)$ of rats before treatment were pooled in the last three figures of this article to make them clearer, but the statistical analyses performed took account of all six groups.

\section{Results}

Food intake

All LPS-treated rats survived. In control rats, LPS treatment induced anorexia throughout the study period (from days 0 to 3). LPS induced anorexia in diabetic rats from days 0 to 1 only. As expected, food intake was similar between LPStreated groups and their respective PF groups (Fig. 1). 


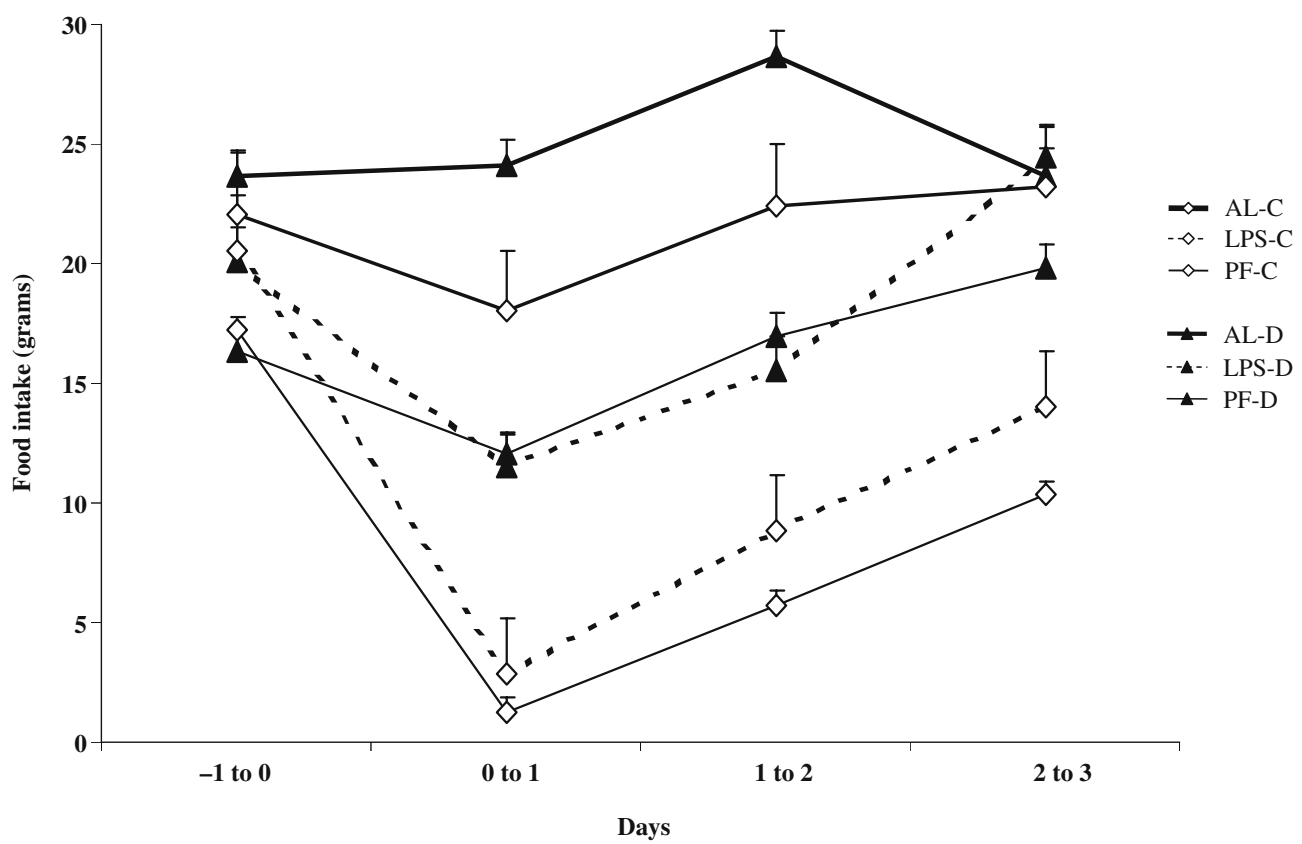

Fig. 1 Food intake (means \pm SEM) in control or obese diabetic ZDF rats. For a description of the various groups, see Materials and methods. Significance of differences: (means \pm SEM, ANOVA plus PLSD Fisher test): day -1 to day 0: AL-C vs PF-C, $p<0.001$; AL-D vs PF-D, $p<0.01$; LPS-D vs PF-D, $p<0.05$; day 0 to day 1 : LPS-C vs AL-C, LPS-D vs AL-D, AL-C vs PF-C, AL-D vs PF-D, $p<0.001$; LPS-C vs LPS-D, $p<0.01$; AL-C vs AL-D, $p<0.05$; day 1 to day 2 :

LPS-D vs LPS-C, $p<0.001$; LPS-C vs AL-C, AL-C vs PF-C, AL-D vs PF-D, $p<0.01$; AL-C vs AL-D, $p<0.05$; day 2 to day 3 : LPS-D vs LPS-C, AL-C vs PF-C, $p<0.001$; LPS-C vs AL-C, $p<0.01$; LPS-D vs PF-D, AL-D vs PF-D, AL-D vs PF-D, AL-D vs AL-C, $p<0.05$. $A L-C$ ad libitum-fed control rats; $A L-D$ ad libitum-fed diabetic rats; $L P S-C$ LPS-treated control rats; $L P S-D$ LPS-treated diabetic rats; $P F-C$ pair-fed control rats; $P F-D$ pair-fed diabetic rats

Relative (i.e. expressed as a percentage of initial weight) body weight

In LPS-C rats, there was a reduction in whole-body weight from days 1 to 3. In LPS-D rats, there was a decrease in body weight from days 1 to 3 . Weight loss was always related to anorexia (no significant difference between PF and LPS groups) (Fig. 2).

\section{Glycaemia and plasma insulin}

On day 3, fasting glycaemia was significantly higher $(p<0.001)$ in the AL-D group than in the AL-C group. Glycaemia was also higher in LPS-D rats than in LPS-C rats $(p<0.001)$. However, glycaemia was lower in the LPS$\mathrm{D}$ group than in the AL-D group $(p<0.001)$. Of note, glycaemia in the PF-D group was not different from the values recorded in the PF-C group (Table 1).

Insulinaemia was higher in the AL-D rats than in AL-C rats $(p<0.001)$, and was also significantly higher in the LPS-D group than the LPS-C group $(p<0.001)$.

Plasma albumin at the end of the experiment

Hypoalbuminaemia followed a similar pattern in LPS-C and LPS-D rats. This alteration was specifically induced by LPS (i.e. non-related to the reduction of food intake)
(LPS-C vs PF-C and AL-C, $p<0.001$; LPS-D vs PF-D and AL-D, $p<0.001$ ) (Table 1).

Tissue weights

The results are reported in Table 1.

\section{Thymus}

In control rats, LPS specifically (i.e. non-related to the reduction of food intake) induced a decrease in thymus weight. In diabetic rats, thymus atrophy was mainly related to anorexia (LPS-D vs AL-D, $p<0.01$ and LPS-D vs PF-D, NS). LPS-mediated thymus involution was lower in diabetic rats than in controls (LPS-D vs LPS-C, $p<0.01$ ).

\section{Spleen}

Endotoxaemia specifically (i.e. non-related to the reduction of food intake) induced splenomegaly in both diabetic and control rats $(p<0.001)$.

\section{EDL muscle}

There was no significant difference in muscle mass between the AL-D and AL-C groups. LPS-linked anorexia 


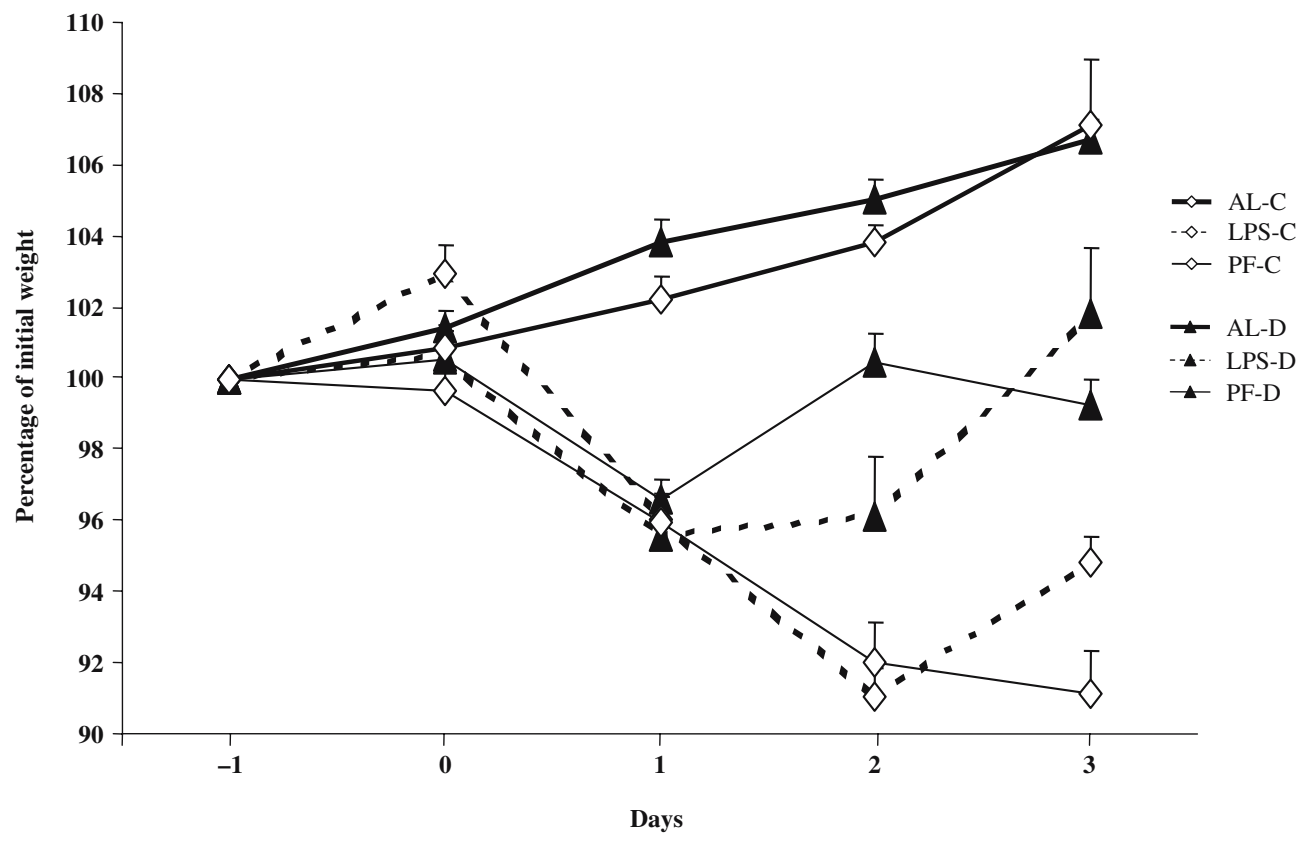

Fig. 2 Relative body weight (percentage of initial body weight, means \pm SEM) of control or obese diabetic ZDF rats. For a description of the various groups, see Materials and methods. Significance of differences: means \pm SEM, ANOVA plus PLSD Fisher test: on day 0: $p<0.05$ AL-C vs LPS-C, LPS D vs LPS-C; $p<0.01$ LPS-C vs PF-C; on day 1: $p<0.001$ AL-C vs PF-C, AL-D vs PF-D, LPS-C vs AL-C, LPS-D vs AL-D; on day 2: $p<0.001$ LPS-C

induced muscle atrophy in control rats (LPS-C vs AL-C, $p<0.05$; LPS-C vs PF-C, NS). Conversely, LPS specifically induced muscle atrophy in diabetic rats (LPS-D vs AL-D, $p<0.01$ and LPS-D vs PF-D, $p<0.05$ ). In addition, muscle atrophy was more significant in diabetic rats than in control rats (LPS-D vs LPS-C, $p<0.05$ ).

\section{Liver}

Liver weight was significantly higher in diabetic rats compared with control rats (AL-D vs AL-C; PF-D vs PF-C; vs AL-C, AL-C vs PF-C, LPS-D vs LPS-C, LPS-D vs AL-D; $p<0.01$ AL-D vs PF-D, LPS-D vs PF-D; on day 3: $p<0.001$ AL-C vs PF-C, AL-D vs PF-D, LPS-C vs AL-C, LPS-D vs LPS-C; $p<0.05$ LPS-D vs AL-D. $A L-C$ ad libitum-fed control rats; $A L-D$ ad libitumfed diabetic rats; $L P S-C$ LPS-treated control rats; $L P S-D$ LPStreated diabetic rats; $P F-C$ pair-fed control rats; $P F-D$ pair-fed diabetic rats

LPS-D vs LPS-C, $p<0.001$ ) with no effect of endotoxaemia (AL-D vs PF-D vs LPS-D: NS).

\section{Jejunum and ileum (data not shown)}

Neither diabetes nor LPS treatment had an effect on jejunum and ileum mass, excluding the LPS-treatmentinduced decrease in ileum mass in diabetic rats (LPS-D vs AL-D, $p<0.05)$.

Table 1 Biochemical features and organ weights (means \pm SEM) in control or obese diabetic ZDF rats

\begin{tabular}{|c|c|c|c|c|c|c|}
\hline & AL-C & $\mathrm{PF}-\mathrm{C}$ & LPS-C & AL-D & PF-D & LPS-D \\
\hline Glycaemia (mmol/l) & $8.40 \pm 0.3$ & $6.5 \pm 0.3$ & $8.3 \pm 0.1$ & $21.6 \pm 1.7^{\mathrm{c}}$ & $7.4 \pm 1.0^{\mathrm{k}}$ & $16.4 \pm 2.3^{\mathrm{h}, \mathrm{k}, \mathrm{l}}$ \\
\hline Insulinaemia $(\mu \mathrm{U} / \mathrm{ml})$ & $67.4 \pm 4.1$ & $10.6 \pm 1.6^{\mathrm{a}}$ & $54.1 \pm 2.4$ & $527.3 \pm 51.2^{\mathrm{c}}$ & $429.0 \pm 68.8^{\mathrm{i}}$ & $681.0 \pm 56.8^{\mathrm{h}, \mathrm{k}, \mathrm{l}}$ \\
\hline Albuminaemia (g/l) & $26.5 \pm 0.2$ & $26.6 \pm 0.3^{\mathrm{c}, \mathrm{e}}$ & $13.8 \pm 0.2$ & $30.9 \pm 0.3$ & $28.9 \pm 0.2$ & $13.1 \pm 0.3^{\mathrm{k}, 1}$ \\
\hline Thymus (mg) & $433 \pm 14$ & $326 \pm 21$ & $250 \pm 26^{\mathrm{c}, \mathrm{d}}$ & $421 \pm 32$ & $358 \pm 22$ & $332 \pm 26^{\mathrm{g}, \mathrm{j}, 1}$ \\
\hline Spleen (mg) & $550 \pm 18$ & $425 \pm 22$ & $831 \pm 43^{\mathrm{c}, \mathrm{e}}$ & $450 \pm 16^{\mathrm{b}}$ & $409 \pm 16$ & $791 \pm 40^{\mathrm{f}, \mathrm{k}, 1}$ \\
\hline EDL (mg) & $147 \pm 5$ & $132 \pm 6$ & $124 \pm 5^{\mathrm{a}}$ & $129 \pm 11$ & $118 \pm 6^{\mathrm{e}}$ & $99 \pm 5^{\mathrm{f}, \mathrm{i}, 1}$ \\
\hline Liver (g) & $11 \pm 0$ & $7 \pm 0$ & $10 \pm 0$ & $17 \pm 1^{\mathrm{c}}$ & $14 \pm 1^{\mathrm{e}}$ & $18 \pm 1^{\mathrm{h}}$ \\
\hline
\end{tabular}

For a description of the groups, see the Materials and methods section: $A L-C$ Ad libitum-fed control rats, $A L-D$ ad libitum-fed diabetic rats, $L P S-C$ LPS-treated control rats, $L P S-D$ LPS-treated diabetic rats, $P F-C$ pair-fed control rats, $P F-D$ pair-fed diabetic rats Significance of differences (ANOVA plus PLSD Fisher test):

a $p<0.05 ;{ }^{\mathrm{b}} p<0.01 ;{ }^{\mathrm{c}} p<0.001$ vs AL-C

${ }^{\mathrm{d}} p<0.01 ;{ }^{\mathrm{e}} p<0.001 \mathrm{vs}$ PF-C

$\mathrm{f}_{p<0.05 ;{ }^{2}}{ }_{p<0.01 ;}{ }^{\mathrm{h}} p<0.001$ vs LPS-C

${ }^{\mathrm{i}} p<0.05,{ }^{\mathrm{j}} p<0.01 ;{ }^{\mathrm{k}} p<0.001$ vs AL-D

${ }^{1} p<0.001$ vs PF-D 
Fig. 3 Cumulative nitrogen balance (means \pm SEM) in control or obese diabetic ZDF rats. For a description of the various groups, see Materials and methods. Day 0 was the day before LPS was injected. Significance of differences (ANOVA plus PLSD Fisher test): $* * * p<0.001$ vs AL corresponding group; ${ }^{*} p<0.05$ vs LPS-C. $A L-C$ ad libitum-fed control rats; $A L-D$ ad

libitum-fed diabetic rats; $L P S-C$ LPS-treated control rats; $L P S-D$ LPS-treated diabetic rats; $P F-C$ pair-fed diabetic rats pair-fed control rats; $P F-D$

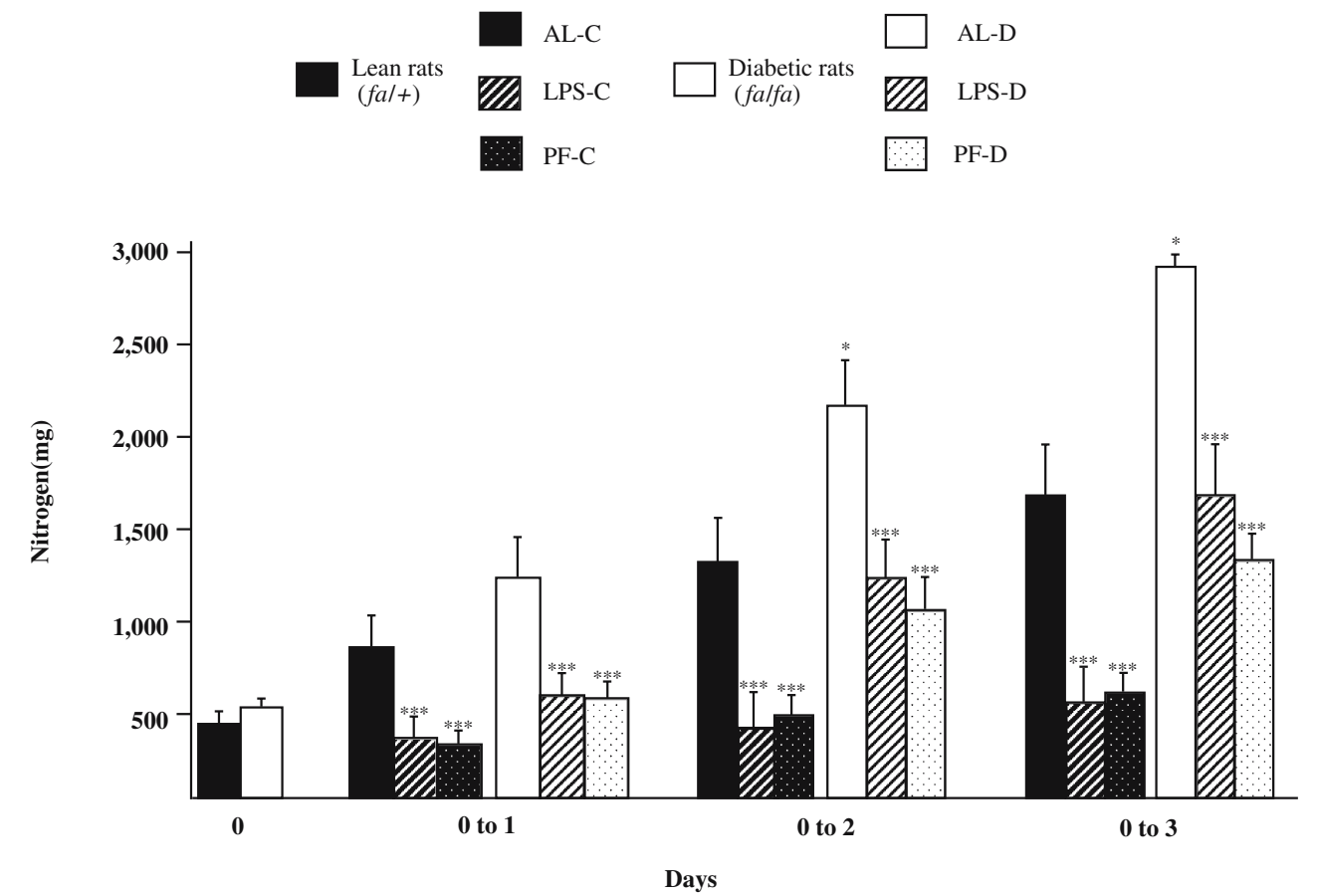

Cumulative nitrogen balance

nitrogen balance was still lower in LPS groups compared with the corresponding AL groups $(p<0.001)$. On days 2 and 3, LPS-D rats showed a higher nitrogen balance compared with LPS-control rats ( $p<0.05$ (Fig. 3).
On day 0 , nitrogen balance was similar in both control and diabetic groups. On day 1, LPS-linked anorexia induced a decrease in nitrogen balance in both control and diabetic rats $(p<0.001)$. From days 0 to 2 , LPS-linked anorexia induced a more pronounced decrease in nitrogen balance in both control and diabetic rats compared with the respective PF untreated rats $(p<0.001)$. From days 0 to 3 , cumulative
Fig. 4 3-Methylhistidine:creatinine ratio (means \pm SEM) from day 0 (day before LPS was injected) to day 3 in control or obese diabetic ZDF rats. For a description of the various groups, see Materials and methods. Significance of differences (means \pm SEM ANOVA plus PLSD Fisher test): day 2 to day $3: * * p<0.01$ vs AL corresponding group, ${ }^{\#} p<0.01$ vs LPS-C. $A L-C$ ad libitum-fed control rats; $A L-D$ ad libitum-fed diabetic rats; $L P S-C$ LPS-treated control rats; $L P S-D$ LPS-treated diabetic rats; $P F-C$ pair-fed control rats; $P F-D$ pair-fed diabetic rats
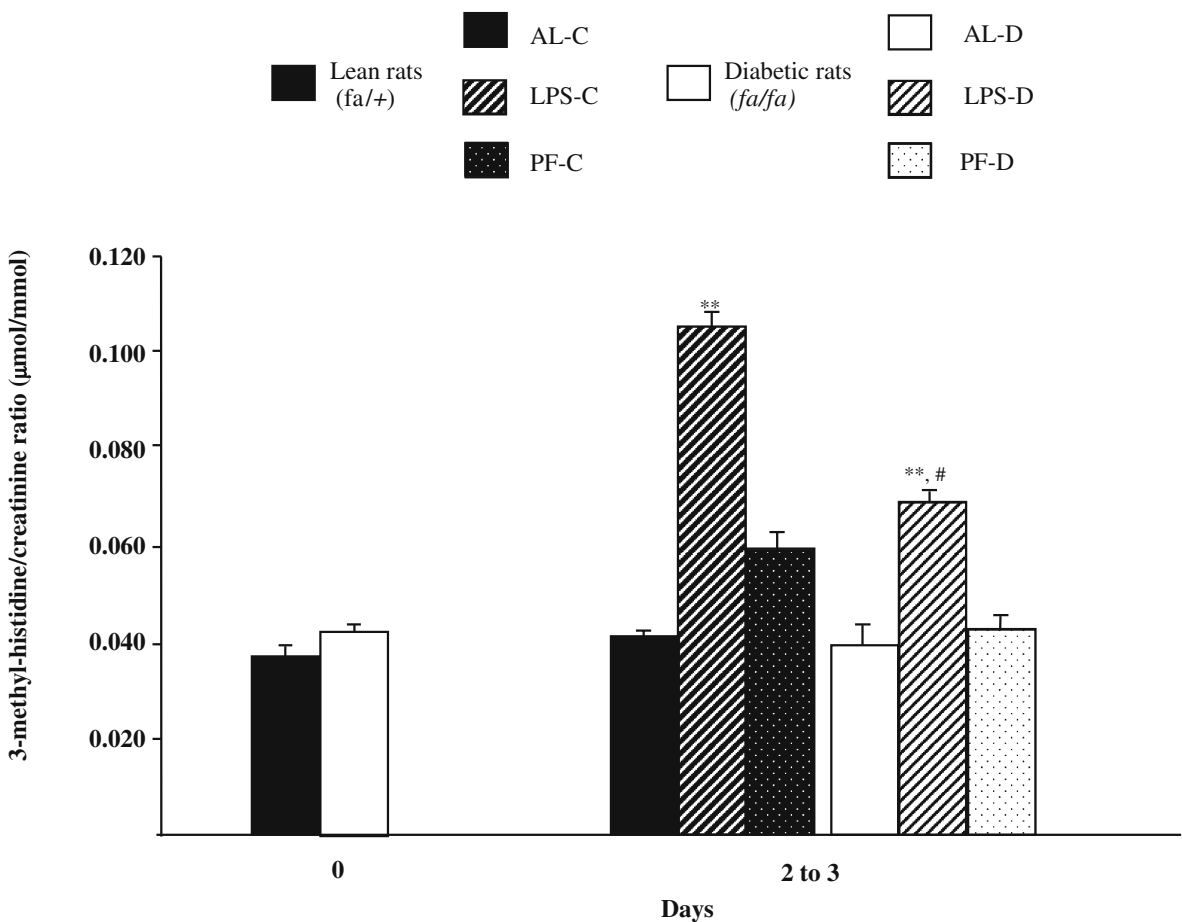
Fig. 5 Urinary NO metabolites (Nox; nitrite and nitrate) in control or obese diabetic ZDF rats (means \pm SEM). For a description of the various groups, see Materials and methods. Significance of differences (ANOVA plus PLSD Fisher test): ${ }^{\#} p<0.01$ vs AL corresponding group; ${ }^{*} p<0.05$, $* * p<0.01$ vs PF corresponding group. $A L-C$ ad libitum-fed control rats; $A L-D$ ad

libitum-fed diabetic rats; $L P S-C$ LPS-treated control rats; $L P S-D$ LPS-treated diabetic rats; $P F-C$ pair-fed control rats; $P F-D$ pair-fed diabetic rats
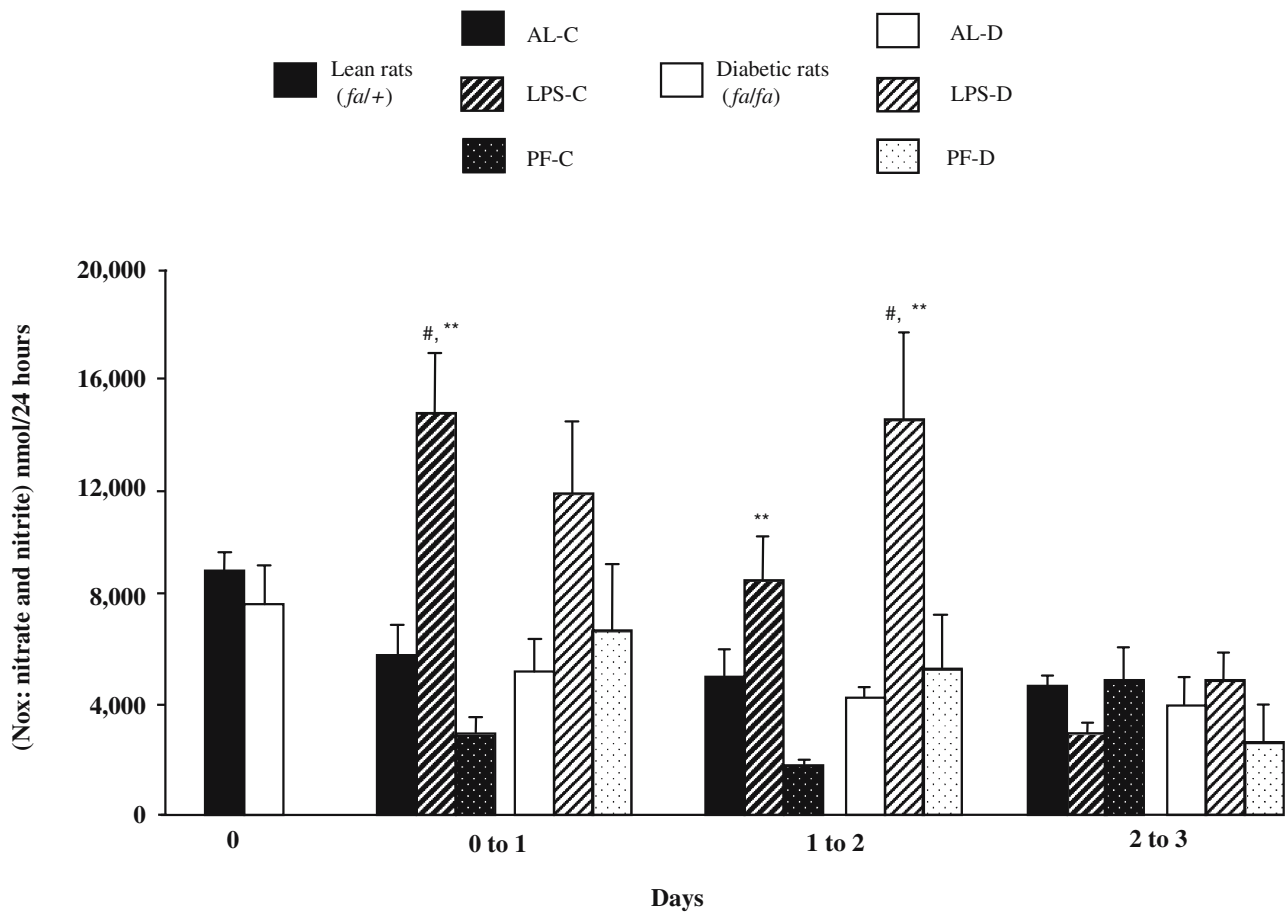

Table 2 Plasma amino acid concentrations (means \pm SEM, $\mu \mathrm{mol} / \mathrm{l}$ ) in control or obese diabetic ZDF rats

\begin{tabular}{|c|c|c|c|c|c|c|}
\hline Amino acid & AL-C & $\mathrm{PF}-\mathrm{C}$ & LPS-C & AL-D & PF-D & LPS-D \\
\hline Arginine & $260 \pm 9$ & $142 \pm 6$ & $300 \pm 5^{\mathrm{a}}$ & $182 \pm 13^{\mathrm{c}}$ & $123 \pm 18$ & $195 \pm 20^{1}$ \\
\hline Aspartate & $13 \pm 1$ & $9 \pm 1$ & $14 \pm 1^{d}$ & $9 \pm 2$ & $9 \pm 2^{\mathrm{m}}$ & $15 \pm 1^{\mathrm{g}}$ \\
\hline Citrulline & $100 \pm 7$ & $72 \pm 7^{b}$ & $78 \pm 4^{\mathrm{a}}$ & $105 \pm 8$ & $94 \pm 3$ & $89 \pm 7$ \\
\hline Cysteine & $38 \pm 2$ & $39 \pm 3^{c}$ & $52 \pm 1^{\mathrm{b}, \mathrm{f}}$ & $36 \pm 2$ & $35 \pm 1$ & $37 \pm 2^{k}$ \\
\hline Glutamate & $78 \pm 6$ & $53 \pm 8$ & $71 \pm 4$ & $131 \pm 5^{\mathrm{c}}$ & $118 \pm 4^{\mathrm{f}}$ & $119 \pm 10^{1}$ \\
\hline Glycine & $333 \pm 67$ & $439 \pm 23$ & $446 \pm 16$ & $194 \pm 15^{\mathrm{c}}$ & $206 \pm 11$ & $206 \pm 14^{1}$ \\
\hline Histidine & $86 \pm 3$ & $76 \pm 3^{b}$ & $82 \pm 1$ & $66 \pm 3^{c}$ & $58 \pm 2^{\mathrm{g}}$ & $63 \pm 2^{1}$ \\
\hline Isoleucine & $112 \pm 5$ & $82 \pm 5$ & $103 \pm 3$ & $152 \pm 3^{c}$ & $85 \pm 8$ & $137 \pm 6^{\mathrm{g}, 1}$ \\
\hline Leucine & $198 \pm 8$ & $143 \pm 8$ & $197 \pm 6$ & $258 \pm 4^{\mathrm{c}}$ & $147 \pm 13$ & $241 \pm 10^{1}$ \\
\hline Proline & $252 \pm 9$ & $116 \pm 5^{\mathrm{c}}$ & $259 \pm 7^{\mathrm{f}}$ & $264 \pm 23$ & $130 \pm 9^{\mathrm{q}, \mathrm{i}}$ & $239 \pm 29$ \\
\hline Taurine & $245 \pm 20$ & $235 \pm 12$ & $246 \pm 12$ & $253 \pm 17$ & $288 \pm 15$ & $281 \pm 29$ \\
\hline Threonine & $256 \pm 7$ & $196 \pm 16^{\mathrm{c}}$ & $259 \pm 5^{\mathrm{e}}$ & $209 \pm 18^{\mathrm{a}}$ & $156 \pm 4^{\mathrm{m}, \mathrm{g}}$ & $197 \pm 16^{\mathrm{k}}$ \\
\hline Tryptophan & $163 \pm 7$ & $153 \pm 20$ & $106 \pm 5^{\mathrm{b}, \mathrm{e}}$ & $125 \pm 6^{\mathrm{a}}$ & $119 \pm 13^{\mathrm{m}}$ & $80 \pm 9^{\mathrm{h}}$ \\
\hline Tyrosine & $114 \pm 3$ & $68 \pm 3^{c}$ & $117 \pm 3^{f}$ & $76 \pm 4^{\mathrm{c}}$ & $57 \pm 12$ & $75 \pm 4^{1}$ \\
\hline Valine & $238 \pm 8$ & $168 \pm 10$ & $240 \pm 7$ & $332 \pm 6^{\mathrm{c}}$ & $195 \pm 14$ & $330 \pm 17^{1}$ \\
\hline
\end{tabular}

For a description of the various groups, see the Materials and methods section: $A L-C$ Ad libitum-fed control rats, $A L-D$ ad libitum-fed diabetic rats, $L P S-C$ LPS-treated control rats, $L P S-D$ LPS-treated diabetic rats, $P F-C$ pair-fed control rats, $P F-D$ pair-fed diabetic rats Significance of differences (ANOVA plus PLSD Fisher test):

${ }_{\mathrm{a}}^{\mathrm{a}} p<0.05 ;{ }^{\mathrm{b}} p<0.01 ;{ }^{\mathrm{c}} p<0.001$ vs AL-C

d $p<0.05 ;{ }^{\mathrm{e}} p<0.01 ;{ }_{p}^{\mathrm{f}} p<0.001$ vs PF-C

${ }^{\mathrm{g}} p<0.05 ;{ }^{\mathrm{h}} p<0.01 ;{ }^{1} p<0.001$ vs AL-D

$\mathrm{j} p<0.05 ;{ }^{\mathrm{k}} p<0.01 ;{ }^{2} p<0.001$ vs LPS-C

${ }^{\mathrm{m}} p<0.05 ;{ }^{\mathrm{n}} p<0.01 ;{ }^{\mathrm{q}} p<0.001$ vs LPS-D 
Table 3 EDL amino acid concentrations (means \pm SEM, nmol/mg of tissue) in control or obese diabetic ZDF rats

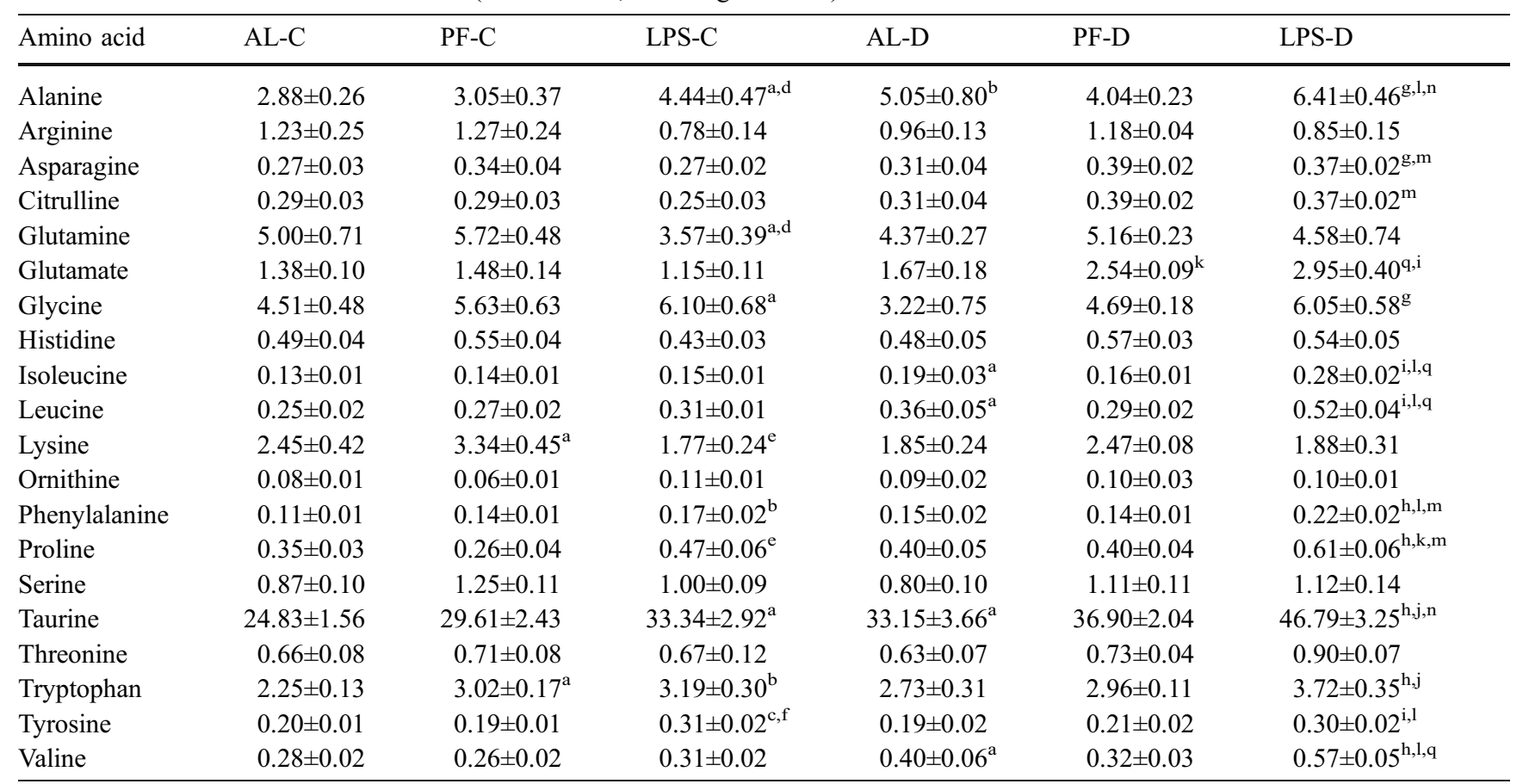

For a description of the various groups, see the Materials and methods section: $A L-C$ Ad libitum-fed control rats, $A L-D$ ad libitum-fed diabetic rats, $L P S-C$ LPS-treated control rats, $L P S-D$ LPS-treated diabetic rats, $P F-C$ pair-fed control rats, $P F-D$ pair-fed diabetic rats Significance of differences (ANOVA plus PLSD Fisher test):

${ }^{\mathrm{a}} p<0.05 ;{ }^{\mathrm{b}} p<0.01 ;{ }^{\mathrm{c}} p<0.001$ vs AL-C

d $p<0.05 ;{ }^{\mathrm{e}} p<0.01 ;{ }_{p}^{\mathrm{f}} p<0.001$ vs PF-C

${ }^{\mathrm{g}} p<0.05 ;{ }^{\mathrm{h}} p<0.01 ;{ }^{1} p<0.001$ vs AL-D

j $p<0.05 ;{ }^{k} p<0.01 ;{ }^{1} p<0.001$ vs PF-D

${ }^{\mathrm{m}} p<0.05 ;{ }^{\mathrm{n}} p<0.01 ;{ }^{\mathrm{q}} p<0.001$ vs LPS-C

Urinary 3-MH: creatinine ratio

From days -1 to 0 , there was no significant difference in 3$\mathrm{MH}:$ creatinine ratio between control and diabetic groups. From days 2 to 3, LPS induced specifically (i.e. non-related to the reduction of food intake) an increase in the 3-MH: creatinine ratio in both control and diabetic rats $(p<0.01)$, with a significantly $(p<0.01)$ higher $3-\mathrm{MH}$ :creatinine ratio in LPS-C than LPS-D rats (Fig. 4).

\section{Urinary NOx}

On day 0 , diabetic rats presented no significant difference in urinary NOx compared with control rats (Fig. 5).

On day 1, urinary NOx levels were increased in LPS-C rats $(p<0.01)$ compared with untreated control and PF-C rats. On day 2, urinary NOx was higher in LPS-D rats compared with untreated diabetic rats and PF-D rats $(p<0.01)$.
Amino acid concentrations

\section{In plasma}

LPS induced a significant increase in arginine, alanine, lysine, cysteine and methionine plasma concentrations in control rats (Table 2).

The diabetic state induced an increase in concentrations of branched-chain amino acids (BCAAs) (valine, leucine and isoleucine) and glutamate plasma levels, and a decrease in arginine, glutamine, histidine, lysine, glycine, threonine, tyrosine and tryptophan plasma levels. LPS treatment did not induce further variation in amino acid plasma levels in the diabetic rats.

\section{In $E D L$}

LPS treatment induced a decrease in muscle glutamine levels $(p<0.05)$ in control rats but not in diabetic rats. The diabetic state induced an increase in BCAA levels 
compared with control rats (AL-D vs AL-C, $p<0.05)$. In diabetic rats, LPS specifically induced an increase in BCAA levels. In control rats, LPS-related anorexia induced a significant increase in phenylalanine levels (LPS-C vs AL-C, $p<0.01$ and LPS-C vs PF-C, NS). In diabetic rats, LPS specifically induced a significant increase in phenylalanine levels (LPS-D vs PF-D and AL-D, $p<0.01$ ) (Table 3).

\section{In jejunum and ileum}

No significant between-group variations in jejunal and ileal amino acid levels were observed (data not shown).

\section{Discussion}

It is well known that obesity associated with type 2 diabetes affects nutritional status [18]. However, the literature contains few data on the metabolic response to stress in obese and diabetic patients. In this study, we aimed to investigate characteristic metabolic alterations in obese and type 2 diabetic rats, and then to evaluate the effect of endotoxaemia. Since LPS administration decreases food intake, we also investigated the effects of food restriction.

\section{Effect of obesity and type 2 diabetes}

As expected, AL-D rats were obese, hyperglycaemic and hyperinsulinaemic when compared with AL-C rats. The results presented here highlight major alterations in amino acid patterns, which have been little studied in the literature to date. Among these alterations, the depletion in plasma glutamine and arginine pools occurring in the type 2 diabetic rats deserves comment. This observed depletion is in agreement with the results of a previous study performed in our laboratory in Zucker rats [19] and with another recent study [13] in the same of ZDF rat model. Interestingly, there is a recent report of hypoargininaemia in diabetic patients [20]. The mechanism underlying arginine depletion in diabetes is not known. On the basis of tracer-based metabolic studies, it has been suggested that arginine disposal and/or dietary arginine intake, rather than de novo arginine synthesis, are the primary regulators of arginine homeostasis [21]. Hence, it can be hypothesised that plasma arginine depletion in ZDF rats is related more to an increase in arginine degradation than to a decrease in arginine supply or de novo arginine synthesis. This hypothesis is supported by evidence that inflammatory stimuli induce catabolic enzymes of arginine such as type I and type II arginases, particularly in the diabetic state, which is associated with low-grade inflammation [21]. In this study, NOx excretion was similar in ZDF rats and in control rats. The balance between inducible NO synthase (NO production) and arginase plays a key role in the regulation of arginine availability. There may be a higher peripheral utilisation of arginine by arginase at the expense of inducible NO synthase. Hypoargininaemia is clearly related to arginine availability, since a recent study showed that an arginine-supplemented diet improved NOx production in the serum of ZDF rats [22]. Our data demonstrated a depletion of glutamine, in accord with a recent study [13] reporting a decrease in plasma concentrations of several gluconeogenic amino acids in ZDF rats. This hypoglutaminaemia may be explained by an increase in gluconeogenic utilisation occurring as a consequence of the insulin resistance [23].

It is also interesting to note that our data on BCAAs are in agreement with another study showing an increase of these amino acids in plasma and muscle in the same model [13]. This pattern was also reported in type 1 diabetes in the streptozotocin diabetic rat model [24]. The increase in BCAA levels in type 1 diabetes has been attributed to the increase in whole-body protein catabolism and hyperphagia [25]. Therefore, this increase in BCAA levels is characteristic of diabetes, whatever the cause [26].

\section{Effect of food restriction}

Food restriction had specific effects in both healthy and diabetic rats, and thus deserves brief comment. In control rats, food restriction (i.e. the PF-C group) led to only minor changes, probably because it was short-lasting. In the diabetic rats, we observed a remarkable normalisation of glycaemia together with a marginal (but significant) decrease in insulinaemia. This suggests that hyperphagia is a major determinant of insulin resistance in this model, as observed in humans [27]. The increase in plasma BCAA levels classically observed in diabetes, as discussed above [26], was no longer observed in food-restricted rats. One study suggested a link between insulin levels and BCAA levels [28]; more recently, a direct relationship was reported between insulin infusion and BCAA levels [29]. However, the link between insulin resistance and BCAA levels needs further investigation. What appears to have occurred in the study is not just related to food restriction, because most essential amino acids other than BCAAs did not decrease.

Different metabolic responses to LPS between control and ZDF rats

An endotoxaemic rat model was used since endotoxaemia may be involved in the pathogenesis of sepsis, and it is known to induce a marked and reproducible hypercatabolic state [30]. LPS was used at a $3 \mathrm{mg} / \mathrm{kg}$ of body weight dose, as in previous studies [10], since it elicits a reproducible catabolic state without lethality. We used the same LPS dose in control and obese rats since we previously found in a separate study that there was no significant difference in 
fat-free mass between control and ZDF rats. The only difference observed was in fat mass, which is obviously higher in ZDF rats. This LPS dosage induces a short-lasting catabolic state $[10,11]$, and the endotoxaemic rats recover 3 days after injury [11]. As reported in other models of stress (e.g. burn, sepsis) [12, 31], LPS administration induced anorexia that lasted throughout the study (i.e. 3 days) in control rats but for only 2 days in diabetic rats. Thus, the use of PF groups allowed us to separately study the effects of food restriction and the specific effects of LPS treatment (see above comments on the specific effects of food restriction). Our data show that the most significant metabolic changes observed in this study in both lean and diabetic rats were specifically related to LPS treatment. In agreement with previous reports, these metabolic changes in response to stress included an alteration in protein metabolism [32], inflammation (as reflected by hypoalbuminaemia) [33], and alterations in immune status (as reflected by thymic involution and spleen hypertrophy, which are a consequence of the redistribution of lymphocytes to the lymphoid organs and the gastrointestinal tract) $[12,34]$. In our work, endotoxaemia led to myofibrillar protein catabolism, since the urinary 3-MH:creatinine ratio increased. As expected, the LPS treatment induced a decrease in nitrogen balance, reflecting net proteolysis in the muscles $[35,36]$. In addition, endotoxaemia induced a higher NOx excretion; indeed it is well-known that LPS is a potent stimulus for NO production, which is the main mediator of microbiocidal actions of macrophages [37].

The diabetic rats showed less catabolism in response to LPS than control animals, since on days 2 and 3 after LPS injection, the diabetic rats showed lower thymic involution and a lower increase in urinary 3-MH:creatinine ratio, and no muscle glutamine depletion, in contrast to the control rats. Indeed, it is known that injury is associated with changes in free amino acid concentrations in both plasma and skeletal muscles $[36,38]$. In particular, a significant decrease in glutamine pools is characteristic of the response to injury [38, 39]. Furthermore, cumulative nitrogen balance was higher in LPS-D rats than in LPS-C. This latter result could be explained by the fact that the ZDF rats were hyperphagic, and demonstrated short-term anorexia in response to stress. However, this hypothesis can be ruled out as the sole explanation, since food intake modulates protein synthesis more than it decreases protein catabolism [40]. Therefore, it would be useful to measure tissue protein synthesis rates using stable isotopes in a future study.

In contrast with other studies [41, 42], the obesity associated with diabetes in our experimental model of stress in ZDF rats appears not to be a cofactor of morbidity and mortality. In the studies cited [41, 42], whole-body protein turnover and protein synthesis were higher in obese traumatised patients than non-obese traumatised patients, and daily muscle catabolism was also higher in the obese patients. The metabolic response of our obese ZDF rats is probably more similar to the fair adaptation of obese subjects to starvation: these subjects had better nitrogen preservation than lean subjects and have been shown to be able to survive longer during hunger strike [43, 44]. The hypothetical 'protective effect' of obesity post-injury may be related to a possible sparing action of fat on protein metabolism [45-47]. In fasted obese patients, an inverse relationship has been shown between urinary nitrogen excretion and level of adiposity in both human and animal models, suggesting a protein-sparing effect of lipids [45]. A study [45] comparing obese subjects with lean subjects showed that, during prolonged starvation, protein loss and percent energy derived from protein oxidation were reduced by more than $50 \%$ in the obese than in the lean subjects. These authors reported an increase in both protein turnover and leucine oxidation in the lean subjects during short-term starvation, whereas there were no significant changes in the obese subjects [45]. One elegant study [46] demonstrated an inverse relationship between leucine oxidation and NEFA levels (and NEFA oxidation) in dogs. In humans, a moderate increase in NEFA (nearly $1 \mathrm{mmol} / \mathrm{l}$ ) also resulted in a $20 \%$ reduction in leucine oxidation [47].

In conclusion, ZDF rats present alterations in their amino acid metabolism patterns, including plasma glutamine and arginine depletion and increased BCAAs. Supplementation with an arginine-rich diet in obese patients should be considered for future study. Interestingly, several parameters tend to show that, under the same stress conditions, the metabolic response of ZDF rats was less severe compared with lean rats, indicating that obesity associated with type 2 diabetes has a possible sparing effect on protein metabolism. However, the underlying mechanisms of this sparing effect require further study.

Acknowledgements This study was supported in part by funds from the French Ministry of Research and Technology (EA 2498) and in part by an unrestricted grant from Nestlé Clinical Nutrition (Noisiel, France). Part of this work was presented at the 26th ESPEN Congress (Lisbon, Portugal, September 2004).

Duality of Interest None of the authors involved in this study had any conflict of interest.

\section{References}

1. Bjorntorp P (1997) Obesity. Lancet 350:423-426

2. Dhatariya K, Sreekumaran Nair K (2004) Diabetes mellitus. In: Cynober L (ed.) Metabolic and therapeutics aspects of amino acids in clinical nutrition. CRC, Boca Raton, Florida, pp 355373

3. Choban PS, Flancbaum L (2000) Nourishing the obese patient. Clin Nutr 19:305-311

4. Biolo G, Toigo G, Ciocchi B et al (1997) Metabolic response to injury and sepsis: changes in protein metabolism. Nutrition 13:52S-57S

5. Van Den Berghe G, Wouters P, Weekers F et al (2001) Intensive insulin therapy in the critically ill patients. N Engl J Med 345:1359-1367

6. Chiolero RL, Tappy L, Berger MM (2003) Nutritional support of obese critically ill patients. Nestle Nutrition Workshop Series. Clinical and Performance Programme 187-200 
7. De Bandt JP (2004) Insulin and the regulation of amino acid catabolism and protein turnover. In: Cynober L (ed.) Metabolic and therapeutics aspects of amino acids in clinical nutrition. CRC, Boca Raton, Florida, pp 355-373

8. Peterson RG, Shaw WN, Neel M-A et al (1990) Zucker diabetic fatty rat as a model for non-insulin-dependent diabetes mellitus. ILAR News 32:16-19

9. Unger RH (1997) How obesity causes diabetes in Zucker diabetic fatty rats. Trends Endocrinol Metab 8:276-282

10. Pernet P, Coudray-Lucas C, Schneid C, Jardel A, Cynober L (2004) Dose dependency of the effect of ornithine alphaketoglutarate on tissue glutamine concentrations and hypercatabolic response in endotoxaemic rats. Br J Nutr 92:627-634

11. Loi C, Osowska S, Neveux N, Darquy S, Cynober L, Moinard C (2005) Effects of an immune-enhancing diet in endotoxemic rats. Nutrition 21:255-263

12. Schlegel L, Coudray-Lucas C, Barbut F, Le Boucher J, Pernet P, Cynober L (1999) Bacterial dissemination, rather than translocation, mediates hypermetabolic response in endotoxemic rats. Crit Care Med 27:1511-1516

13. Wijekoon EP, Skinner C, Brosnan ME, Brosnan JT (2004) Amino acid metabolism in the Zucker diabetic fatty rat: effects of insulin resistance and of type 2 diabetes. Can J Physiol Pharmacol 82:506-514

14. Young VR, Munro HN (1978) Ntau-methylhistidine (3methylhistidine) and muscle protein turnover: an overview. Fed Proc 37:2291-2300

15. Blanc MC, Neveux N, Laromiguiere M, Berard MP, Cynober L (2000) Evaluation of a newly available biochemical analyzer: the Olympus AU 600. Clin Chem Lab Med 38:465-475

16. Grimble GK, West MF, Acuti AB et al (1988) Assessment of an automated chemiluminescence nitrogen analyzer for routine use in clinical nutrition. JPEN J Parenter Enteral Nutr 12:100-106

17. Green LC, Wagner DA, Glogowski J, Skipper PL, Wishnok JS, Tannenbaum SR (1982) Analysis of nitrate, nitrite, and $\left[{ }^{15} \mathrm{~N}\right]$ nitrate in biological fluids. Anal Biochem 126:131-138

18. Unger RH, Orci L (2001) Diseases of liporegulation: new perspective on obesity and related disorders. FASEB J 15:312321

19. Blanc MC, Moinard C, Béziel A, Darquy S, Cynober L, De Bandt JP (2004) Arginine and glutamine availability and macrophage functions in the obese insulin-resistant Zucker rat. J Cell Physiol 202:153-159

20. Long K, Tayek JA (1999) Are gluconeogenic amino acids reduced in obese and non-obese type 2 diabetic patients (DM-2) compared to weight similar normal volunteers? Diabetes 48 : A460-A461

21. Morris SM J (2000) Regulation of arginine availability and its impact on NO synthesis. In: Ignarro JL (ed) Nitric oxide: biology and pathobiology. Academic, San Diego, California, pp 187-197

22. Fu WJ, Haynes TE, Kohli R et al (2005) Dietary l-arginine supplementation reduces fat mass in Zucker diabetic fatty rats. $\mathrm{J}$ Nutr 135:714-721

23. Stumvoll M, Perriello G, Nurjhan N et al (1996) Glutamine and alanine metabolism in NIDDM. Diabetes 45:863-868

24. Brosnan JT, Man KC, Hall DE, Colbourne SA, Brosnan ME (1983) Interorgan metabolism of amino acids in streptozotocindiabetic ketoacidotic rat. Am J Physiol 244:E151-E158

25. Rodriguez T, Alvarez B, Busquets S, Carbo N, Lopez-Soriano FJ, Argiles JM (1997) The increased skeletal muscle protein turnover of the streptozotocin diabetic rat is associated with high concentrations of branched-chain amino acids. Biochem Mol Med 61:87-94

26. Nair KS, Short KR (2005) Hormonal and signaling role of branched-chain amino acids. J Nutr 135:1547S-1552S
27. Manco M, Mingrone G (2005) Effects of weight loss and calorie restriction on carbohydrate metabolism. Curr Opin Clin Nutr Metab Care 8:431-439

28. Marchesini G, Bianchi GP, Vilstrup H, Capelli M, Zoli M, Pisi E (1991) Elimination of infused branched-chain amino-acids from plasma of patients with non-obese type 2 diabetes mellitus. Clin Nutr 10:105-113

29. Freyse EJ, Fischer U, Knospe S, Ford GC, Nair KS (2006) Differences in protein and energy metabolism following portal versus systemic administration of insulin in diabetic dogs. Diabetologia 49:543-551

30. Lasnier E, Coudray-Lucas C, Le Boucher J, Jardel A, Cynober L (1997) Ornithine $a$-ketoglutarate counteracts thymus involution and glutamine depletion in endotoxemic rats. Clin Nutr 77:197-200

31. Breuille D, Voisin L, Contrepois M, Arnal M, Rose F, Obled C (1999) A sustained rat model for studying the long-lasting catabolic state of sepsis. Infect Immun 67:1079-1085

32. Cooney RN, Kimball SR, Vary TC (1997) Regulation of skeletal muscle protein turnover during sepsis: mechanisms and mediators. Shock 7:1-16

33. Ballmer PE (2001) Causes and mechanisms of hypoalbuminaemia. Clin Nutr 20:271-273

34. Abumrad N, Barbul A (2003) The use of arginine in clinical practice. In: Cynober L (ed.) Metabolic and therapeutics aspects of amino acids in clinical nutrition. CRC, Boca Raton, Florida, pp 355-373

35. Long CL, Birkhahn RH, Geiger JW, Betts JE, Schiller WR, Blakemore WS (1981) Urinary excretion of 3-methylhistidine: an assessment of muscle protein catabolism in adult normal subjects and during malnutrition, sepsis, and skeletal trauma. Metabolism 30:765-776

36. Minet-Quinard R, Moinard C, Walrand S et al (2000) Induction of a catabolic state in rats by dexamethasone: dose or time dependency? JPEN J Parenter Enteral Nutr 24:30-36

37. Mills CD (2001) Macrophage arginine metabolism to ornithine/ urea or nitric oxide/citrulline: a life or death issue. Crit Rev Immunol 21:399-426

38. Austgen TR, Chakrabarti R, Chen MK, Souba WW (1992) Adaptive regulation in skeletal muscle glutamine metabolism in endotoxin-treated rats. J Trauma 32:600-606

39. Parry-Billings M, Leighton B, Dimitriadis G, de Vasconcelos PR, Newsholme EA (1989) Skeletal muscle glutamine metabolism during sepsis in the rat. Int J Biochem 21:419-423

40. Darmaun D (1999) Role of nutrients in the regulation of in vivo protein metabolism in humans. Acta Paediatr Suppl 88:92-94

41. Jeevanandam M, Ramias L, Schiller WR (1991) Altered plasma free amino acid levels in obese traumatized man. Metabolism $40: 385-390$

42. Jeevanandam M, Young DH, Schiller WR (1991) Obesity and the metabolic response to severe multiple trauma in man. J Clin Invest 87:262-269

43. Forbes GB, Drenick EJ (1979) Loss of body nitrogen on fasting. Am J Clin Nutr 32:1570-1574

44. Leiter LA, Marliss EB (1982) Survival during fasting may depend on fat as well as protein stores. JAMA 248:2306-2307

45. Elia M, Stubbs RJ, Henry CJ (1999) Differences in fat, carbohydrate, and protein metabolism between lean and obese subjects undergoing total starvation. Obes Res 7:597-604

46. Tessari P, Nissen SL, Miles JM, Haymond MW (1986) Inverse relationship of leucine flux and oxidation to free fatty acid availability in vivo. J Clin Invest 77:575-581

47. Beaufrere B, Chassard D, Broussole C, Riou JP, Beylot M (1992) Effects of $d$-beta-hydroxy-butyrate, long and medium chain triglycerides on leucine metabolism in man. Am J Physiol 262:E268-E274 